\title{
SITUATIONS AND INTERVALS ${ }^{1}$
}

\author{
Rebecca J. Passonneau \\ Paoli Research Center, UNISYS Defense Systems \\ P.O.Box 517 \\ Paoli, PA 19301 USA
}

\begin{abstract}
The PUNDIT system processes natural language descriptions of situations and the intervals over which they hold using an algorithm that integrates aspect and tense logic. It analyses the tense and aspect of the main verb to generate representations of three types of situationsstates, processes and events- and to locate the situations with respect to the time at which the text was produced. Each situation type has a distinct temporal structure, represented in terms of one or more intervals. Further, every interval has two features whose different values capture the aspectual differences between the three different situation types. Capturing these differences makes it possible to represent very precisely the times for which predications are asserted to hold.
\end{abstract}

\section{Introduction}

This paper describes a semantics of situations and the intervals over which they hold that is neither situation semantics (Barwise and Perry, 1983) nor interval semantics (Dowty, 1979, 1982, 1986; Taylor, 1977). It is unfortunately difficult to avoid the overlap in terminology because what will be described here shares certain goals and assumptions with each. The concerns addressed here, however, arise from the computational task of processing references to situations in natural language text in order to represent what predicates are asserted to hold over what entities and when.

Situation as used here pertains to the linguistic means for referring to things in the

\footnotetext{
'This work was supported by DARPA under contract No0014-85-C-0012, administered by the Office of Naval Research. APPROVED FOR PUBLIC RELEASE, DISTRIBUTION UNLIMITED.
}

'Formerly SDC-A Burroughs Company. world, i.e., to sentences or predications. More specifically, situation is the superordinate category in Mourelatos' typology of aspectual classes of predications, schematised in Fig. 1.

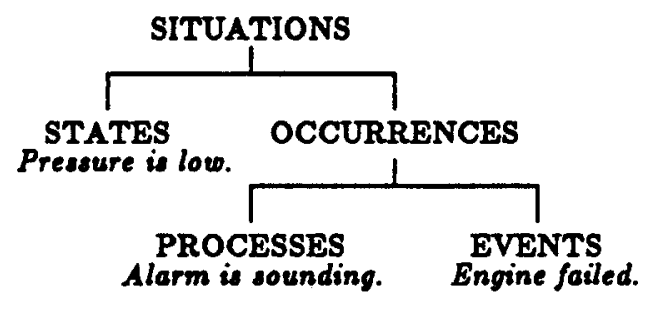

Fig. 1: Mourelatos' typology of situations

The PUNDIT text-processing system ${ }^{3}$ processes references to situations using an algorithm that integrates tense logic (Reichenbach, 1947) with aspect, or what Talmy (1985) calls the pattern of distribution of aetion through time. This paper describes how PUNDIT represents the temporal structure of three types of situations, namely states, processes and events, and how these situations are located in time.

\section{Problems in Computing Appropriate Representations}

The critical problems in the semantic analysis of references to situations and their associated times are: 1) language encodes several different kinds of temporal information, 2) this information is distributed in many distinct linguistic elements, and finally, 3) the semantic contribution of many of these elements is context-dependent and cannot be computed without looking at co-occurring elements.

\footnotetext{
'PUNDIT is an acronym for Prolog UNderatands and Integrates Text. It is modular system, implemented in Quintur Prolog, with distinct syntactic, semantic and pragmatic components (cP. Dahl et al., 1987).
} 
These problems have been addressed as follows. A decision was made to focus on the kinds of temporal information embodied in the verb and its categories of tense, taxis and grammatical aspect, 5 and to temporarily ignore other kinds of temporal information. Computation of this information was then divided into two relatively independent tasks, with appropriate information passed between the modules performing these tasks in order to accommodate contextdependencies. The first task, carried out by Module 1 , makes use of the aspectual information in the verb phrase (lexical and grammatical aspect) to determine the situation type being referred to and its temporal structure. An abstract component of temporal structure, referred to as the event time (following Reichenbach, 1947), serves as input to Module 2, where the deictic information in the verb phrase (tense and taxis) is used to compute temporal ordering relations, i.e., where the situation is located with respect to the time of text production. Section $\$ 3$ outlines the general goals for computing temporal structure and $\$ 4$ describes in detail how it is computed. Then $\$ 5$ briefly illustrates how the event time which Module 1 passes to Module 2 simplifies the interaction of tense and aspect.

\section{Goals for Representing Situations}

The goal in generating representations of the temporal structure of situations was to closely link the times at which situations are said to hold with the lexical decompositions of the predicates used in referring to them. The decompositions encode aspectual information about the situation types which is used in determining what type of situation has been referred to and what its temporal structure is. Distinct components of the semantic decompositions correspond to different features of the intervals with which they are associated. As $\S 4$ will demonstrate, the interpretation of these components of temporal meaning depends on the interaction between lexical and grammatical aspect.

\footnotetext{
'Taxis (Jakobson, 1957) refers to the semantic effeet of the presence or absence of the perfect auxiliary.

'Aspect is both part of the inherent meaning of a verb (lexical aspect) and also eignalled by the presence or absence of the progressive auffix -ing (grammatical aspect).

'E.c., rate (civen by adverbs like repidly), "patteras of frequency or habituation", and 20 on (cf. Mourelatos, 1981).
}

This approach to the compositional semantics of temporal reference is similar in spirit to interval semantics. Interval semantics captures the distinct temporal properties of situations by specifying a truth conditional relation between a full sentence and a unique interval (Dowty, 1979,1986 ). This is motivated by the observation that the aspectual type of a sentence depends simultaneously on the aspectual class of a particular lexical item, its tense, taxis and grammatical aspect, and the nature of its arguments (cf. Mourelatos, 1981; note that the latter factor is not handled here).

The goal of PUNDIT's temporal analysis is not simply to sort references to situations into states, processes and events, but more specifically to represent the differences between the three types of situations by considering in detail the characteristics of the set of temporal intervals that they hold or occur over (Allen, 1983, p. 132). Thus, instead of specifying truth conditional properties of sentences, the temporal semantics outlined here specifies what property of an interval is entailed by what portion of the input sentence, and then compositionally constructs a detailed representation of a state, process or event from the interrals and their associated properties.

\subsection{Intervals and Their Features}

Each situation type has a distinct temporal structure comprised of the interval or intervals over which it holds. Two features are associated with each interval, kinesis and boundedness. Very briefly, kinesis pertains to the internal structure of an interval, or in informal terms, whether something is happening within the interval. Boundedness pertains to the way in which an interval is located in time with respect to other times, e.g., whether it is bounded by another interval.

\section{Part One of the Algorithms Computing} Temporal Structure

The input used to compute the temporal structure of a situation consists of the grammatical aspect of the verb, that is, whether it is progressive, and the decomposition produced by PUNDIT's semantic interpreter (Palmer et al., 1986). The lexical decompositions employed by PUNDIT (Passonneau, 1986b) not only represent the predicate/argument structure of verbs, but in addition, following the example of Dowty's aspect 
calculus (1979), they represent a verb's inherent temporal properties, or lexical aspect. ${ }^{7}$ In PUNDIT's lexical entries, there are three values of lexical aspect corresponding to the three types of situations. Four of the six possible combinations of grammatical and lexical aspect are temporally distinct. This section will go through the four cases one by one.

\subsection{States}

The following conditional statement summarizes the first of four cases of temporal structure. The antecedent specifies the necessary input condition, the first clause of the consequent specifies the situstion type, the second specifies the kinesis of its associated interval and the third specifies its boundedness.

\section{IF Lexical Aspect=stative}

THEN Situation is a state

AND its Time Argument is a period

AND this period is unbounded

As shown here, if the lexical aspect of a predication is stative, its grammatical aspect is irrelevant. The justification for ignoring grammatical aspect in the context of lexical stativity appears at the end of this section.

A state is defined as a situation which holds for some interval that is both static and unbounded. Example 1) illustrates a typical reference to a state situation along with its semantic decomposition. Note that the lexical head of the verb phrase is the adjective low.

1) The pressure was low.

low(patient([pressure1]) ${ }^{8}$

As in Dowty's aspect calculus (1979), the decompositions of stative predicates consist of semantic predicates with no aspectual operators or connectives. Computing the temporal structure associated with 1) means finding a single interval with the appropriate features of kinesis and boundedness to associate with the stative predicate low(patient(X)).

\footnotetext{
The literature on aspectual elasses of verbs provides a variety of diagnostics for defermining the inherent aspect of verbs (cf. Vendlez, 1967; Dowty, 1979).

'PUNDIT's currest application is to process short messages texts called CASREPS (CASualty REPorta) which describe Navy equipment failurea. The arguments in the decompositions, e.c., [pressurel], are unique identiflers of the entities denoted by the surface noun phrabes. They are creat-
}

Kinesis of states. A static interval is temporally homogeneous. With respect to the relevant predication, there is no change within the interval; consequently, any subinterval is equivalent to any other subinterval. Thus, a static interval is defined much as stative predications are defined in interval semantics:

An interval $I$ associated with some predication $\psi$ is static iff it follows from the truth of $\psi$ at $I$ that $\psi$ is true at all subintervals of I (cf. Dowty, 1986, p. 42).

Situations are represented as predicates identifying the situation type (e.g., state). The situation denoted by 1) would be represented as follows:

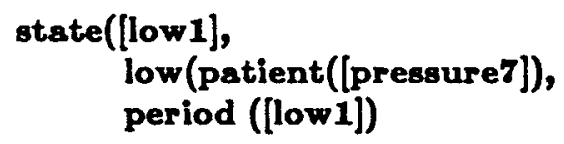

The three arguments are: the unique identifier of the situation (e.g., [low1]), the semantic decomposition, and the time argument (e.g., period ([low1])). The same symbol (e.g.,[low1]) identifies both the sitaation and its time argument because it is the actual time for which a situation holds which uniquely identifies it. ${ }^{\circ} \mathrm{A}$ period time argument in the context of a state predicate always represents a static interval.

Boundedness of states. The intervals associated with states are inherently unbounded. A temporal bound can be provided by an appropriate temporal adverbial (e.g., The pressure was normal watil the pump seized), ${ }^{10}$ but here we consider only the temporal semantics specified by the rerb form itself. When an unbounded interval is located with respect to a particular point in time, it is assumed to extend indefinitely in both directions around that time. In 1), at least part of the interval for which the predication low(patient([pressure1])) is asserted to hold is located in the past. However, this interval may or may not end prior to the present. The unbounded property of the interval can be illustrated more precisely by examining the relationship between the predication and the temporal adverbial

ed by PUNDIT': reference resolution component (Dahl, 1986).

'Though a situation is something quite different for Barwise and Perry (1983), they take a similar view of the role of a particular apace-time location in tokenising a situation type (cl. eap. pp. 51fi).

1OIn general, temporal adverbiale can modify an exinting component of temporal structure or add components of temporal strueture. 
modifying it in example 2):

2) The pressure was low at 08:00.

This sentence asserts that the state of low(patient([pressure1])) holds at 08:00 and possibly prior and subsequent to 08:00. That is, the sentence would be true if the pressure were low for only an instant coincident with 08:00, but it is not asserted to hold only for that instant. This is captured by defining the interval as unbounded.

A situation representation does not itself indicate the boundedness of its period time argument. Instead, this feature is passed as a parameter to the component which interprets tense and taxis (cf. §5).

As will be shown in the following section, the progressive assigns the features active and unbounded to non-stative verbs. But with stative verbs, the progressive contributes no temporal information. Inability to occur with the progressive has in fact been cited as a diagnostic test of statives, but as Dowty notes (1979), there is a class of progressives which denotes locative states (e.g., The socks are lying under the bed). Such statives occur in PUNDIT's current application domain in examples like the following sentence fragment:

3) Material clogging strainer.

A complete discussion of the interaction between progressive grammatical aspect and stative lexical aspect would have to address cases in which the progressive contributes non-temporal information (cf. Smith, 1983). However, these issues are not pertinent to the computation of temporal structure.

\subsection{Temporally Unbounded Processes}

The second case of temporal structure involves progressive uses of non-stative verbs, i.e., process or transition event verbs.

IF Lexical Aspect $\neq$ stative

AND Grammatical Aspect=progressive

THEN Situation is a procese

AND its Time Argument is a period

AND this period is unbounded

In this case and the two subsequent ones, both lexical and grammatical aspect are relevant input.

Processes are situations which hold over active intervals of time. ${ }^{11}$ Active intervals can be unbounded or unspecifled for boundedness, depending on the grammatical aspect of the predication. The two possible temporal structures associated with processes are discussed in this and the following section.

Example 4) illustrates a typical predication denoting a temporally unbounded process along with its semantic decomposition.

4) The alarm was sounding. DO(sound(actor([alarm4])))

DO is an aspectual operator identifying a decomposition as a process predicate (cf. Dowty, 1979). ${ }^{12}$ As with statives, computing the temporal structure for sentences like 4) involves finding a single interval to associate with the semantic decomposition.

Kinesis of processes. The presence of a DO operator in a decomposition indicates that the interval for which it holds must be active. Active and static intervals contrast in that change occurs within an active interval with respect to the relevant predication. For example, for any interval for which DO(sound(actor([alarm4]))) is true, the [alarm4] participant must undergo changes that qualify as sounding, and must continue to do so throughout the interval. As Mourelatos (1981) has pointed out, process predicates vary regarding how narrowly one can subdivide such intervals and still recognize the same process. Dowty has used this threshold of granularity as the defining characteristic of process sentences, and it is borrowed here to define active intervals:

An interval $I$ associated with some predication $\psi$ is active if it follows from the truth of $\psi$ at $I$ that $\psi$ is true at all subintervals of $I$ down to a certain limit in size.

As the process representation for 4) illustrates, processes and states are represented similarly.

process([sound3], low(patient([alarm7]), period ([sound3])

"The distinction between statie and active intervals is useful for interpreting manner adverbials indicating rate of change. Since stative predications denote the absence of change over time, they cannot be modified by rate adverbials.

${ }^{13}$ Because the aspectual operator DO always has an actor semantic role associated with it, PUNDIT's semantic decompositions actually omit DO and use the presence of the actor role to identify process predicates. 
The situation predicate identifies the situation type as a process. Note that a period time argument in the context of a process predicate indicates an active interval.

The rule given above specifies that transition event verbs in the progressive also denote temporally unbounded processes (cf. 5).

5) The engineer is installing the oil filter. cause(DO(install(agent([engineer 3]))),

BECOME(installed(theme([filter 4]), location(X))))

The cause predicate in the decomposition of install indicates that it is a causative verb, and the BECOME operator that its lexical aspect is transition event. This aspectual class is a heterogeneous one, but in general, transition event verbs are temporally more complex than stative or process verbs, and have a correspondingly more complex relation between their semantic decompositions and temporal structure. Consequently, the discussion of the treatment of progressive transition event rerbs is postponed until after the function of the aspectual operator BECOME has been explained.

Boundedness. In 6), the interval associated with the alarm sounding is unbounded. It bears the same relationship to the at adverbial phrase modifying the predication as does the static inter$\mathrm{val}$ in 2) above, repeated here as 7).

6) The alarm was sounding at 08:00.

7) The pressure was low at 08:00.

This similarity between statives and progressives has led Vlach (1981) to identify them with each other. Here, the commonality among sentences like 1), 2), 4) and 6) is captured by associating the feature value unbounded both with stative lexical aspect and with progressive grammatical aspect. The differences between the predications in 6) and 7), which show up in the types of modification and anaphoric processes to which such predications are susceptible, are encapsulated in their contrasting values of kinesis (cf. fn. 11 above).

\subsection{Temporally Unspecified Processes}

The third case of temporal structure accounts for the differences between sentences like 4), having a process verb in the progressive, and 8), where the process verb is non-progressive.
8) The alarm sounded.

The differences, which will be explained below, are captured in the following rule indicating that the active interval for which the predication is said to hold is unspecified for boundedness.

IF Lexical Aspect=process

AND Grammatical Aspect=non-progressive

THEN Situation is a process

AND its Time Argument is a period

AND this period is unspecified

Again, the parameter indicating that the interval associated with 8) is unspecified gets passed to Module 2 which interprets tense and taxis. In every other respect, the analysis of the temporal structure associated with 8) resembles that for 4).

A comparison of progressive and nonprogressive process verbs in the context of an at adverbial phrase illustrates the relative indeterminacy of the non-progressive use. In the context of the progressive process verb in 6), the clock time is interpreted as falling within the active interval of sounding but in 9), where the verb is not progressive, 08:00 can be interpreted as falling at the inception of the process or as roughly locating the entire process.

9) The alarm sounded at 08:00.

Non-progressive process verbs exhibit a wide variation with respect to what part of the temporal structure is located by tense (Passonneau, 1986a). The influencing factors seem to be pragmatic in nature, rather than semantic. The solution taken here is to characterise the event time of such predications as having an unspecifled relation to the active interval associated with the denoted process.

\subsection{Transition Events}

As mentioned in the previous section, the temporal structure of transition events is more complex than that of states or processes. Correspondingly, the rule which applies to this case has more output conditions.

IF Lexical Aspect=transition event

AND Grammatical Aspect=non-progressive

THEN Situation=event

AND Time Argument=moment

AND this moment culminates an interval associated with a process

AND this moment introduces an interval associated with a state or process 
A transition event is a complex situation consisting of a process which culminates in a transition to a new state or process. Its temporal structure is thus an active interval followed by-and bounded by-a new active or static interval. The new state or process comes into being as a result of the initial process. ${ }^{\text {is }}$

As in Dowty (1986), both Vendler's achievements and his accomplishments collapse into one class, vir., transition events. That is, achievements

are those kinesis predicates which are not only typically of shorter duration than accomplishments, but also those which we do not normally understand as entailing a sequence of sub-events, given our usual every-day eriteria for identifying the events named by the predicate (Dowty, 1986, p. 43).

Causative verbs, in which the action of one participant results in a change in another participant, are typical accomplishment verbs.

10) The pump sheared the drive shaft. cause(DO(shear(agent([pump6]))), BECOME (Bheared(patient([shaft6]))))

Sentence 10) asserts that a process in which the pump participated (ohearing) caused a change in the drive shaft (being sheared). Note that the decomposition explicitly indicates a causal relation between two conjoined predicates, one representing an activity perfomed by an agent, and the other representing the resulting situation. BECOME serves as the aspectual operator for marking transition event decompositions. The argument to BECOME constitutes the semantic decomposition of the new state or process arising at the culmination of the event.

Non-causative verbs can also denote transition events. With inchoatives, the same entity participates in both the initial process and the resulting situation denoted by the predication.

11) The engine failed.

DO(fail(agent([engine1]))),

BECOME(failed(patient([engine1])))

In 11), an engine is said to participate in some process (failing) which culminates in a new state (e.g., being inoperative). The semantic

${ }^{12} A$ otate may be a necessary precondition for a certain change to occur, but since atatea are defined by the absence of change, or negative kinenis, they are inherently incapable of generating new situations. decompositions used in PUNDIT do not explicitly represent the initial processes involved in transition events because they are completely predictable from the presence of the BECOME operator. But both conjuncts are shown here to illustrate that computing the temporal structure of a transition event situation requires finding two intervals, one associated with the initial process predicate (e.g., DO(fail(agent([engine1]]))) and the other with the predicate for the resulting situation (e.g., failed(patient([engine1]))).

As indicated in the rule for this case, the temporal structure also includes a moment of transition between the two intervals, i.e., its transition bound. Since a transition event is one which results in a new situation, there is in theory a point in time before which the new situation does not exist and subsequent to which it does. A transition bound is a theoretical construct not intended to correspond to an empirically determined time. In fact, it should be thought of as the same kind of boundary between intervals implied by Allen's meets relation (Allen, 1983; 1984, esp. p. 128). However, it is a convenient abstraction for representing how transition events are perceived and talked about.

We can now return to the question of the interpretation of progressive transition event verbs. In the context of a decomposition with a BECOME operator, the progressive is constrained to apply to the predicate corresponding to the initial process, i.e., the predicate denoting the portion of a transition event prior to the moment of transition. Computing the temporal structure for the progressive of install in 12), for example, involves generating a single active, unbounded interval for which the predication DO(agent([engineer 3$])$ ) holds:

12) The engineer is installing the oil filter. cause(DO(agent([engineer 3]),

$$
\begin{array}{r}
\text { BECOME(installed(theme }([\text { fflter4]), } \\
\text { location(X)))) }
\end{array}
$$

In this context, the remainder of the semantic decomposition denotes what the person reporting on the event assumes to be the eventual culmination of the process referred to as installing.

Kinesis. Examples 13) and 14) illustrate two types of transition events, one resulting in a new state, and one resulting in a new process. As illus- 
trated, ${ }^{14}$ transition events are represented as complex situations in which an event with a moment time argument results in a new state or process:

13) The lube oil pump has seised.

event([seise1], BECOME(seired(patient([pump1]))), moment([seise1])

state([Beise2], seised(patient([pump1])), period([Beise2])

starts(moment([seise1]),period([seise2])

14) The engine started.

event([start1],

BECOME(operating(actor([engine1]))), moment ([start1])

process([otart2], operating(actor([engine1])), period([start2])

starts(moment([start1]),period([start2])

The starts relation indicates that a transition bound (e.g., moment([seise1])) is the onset of the interval (e.g., period([Beise2])) associated with the situation resulting from a transition event.

Boundedness. An important role played by the transition bound is that it serves as the temporal component of transition events for locating them with respect to other times. For example, the sentence in 15) asserts that the moment of transition to the new situation coincides with the clock time of 8:00.

15) The engine failed at 8:00.

The status of the engine prior to $8: 00$ is asserted to be different from its status afterwards.

5. Part Two of the Algorithms Temporal Ordering Relations

PUNDIT employs a Reichenbachian analysis of tense which temporally locates situations in terms of three abstract times: the time of the situation (event time), the time of speech/text production (speech time), and the time with

\footnotetext{
14 At present, PUNDIT explicitly represents only two components of transition event predications: the moment associated with an event of becoming, and a period associated with a resulting situation. This representation has been found to be adequate for the current application. The omission of the first interval is purely a matter of practical convenience, but could easily be represented should the need arise.
}

respect to which relational adverbials are interpreted (reference time). Reichenbach (1947) did not distinguish between the temporal structure of a situation and its event time. In PUNDIT, event time is a carefully defined abstract component of temporal structure in terms of which ordering relations are specified. It is determined on the basis of boundedness, and is always represented as a dimensionless moment.

\subsection{Event Time}

The three values of boundedness outlined above correspond to three possible relations of event time to a time argument. Examples 16) through 18) illustrate these relations. If an interval is unbounded, its event time is represented as an arbitrary moment included within the period time argument:

16) The pressure is low.

Boundedness: unbounded

Event time: $M_{1}$ such that

$$
\text { includes(period([low1]),moment } \left.\left(\left[M_{1}\right]\right)\right)
$$

For an interval unspecified for boundedness the event time has a non-committal relation to the interval, i.e., it may be an endpoint of or included within the period time argument:

17) The alarm sounded.

Boundedness: unspecified

Event time: $M_{1}$ such that

has(period([sound1]),moment $\left.\left.\left(\mathbb{M}_{1}\right]\right)\right)$

The moment time argument of a transition event is ideatical to its event time. Identity, or the lack of referential distinctness, is handled through Prolog unification.

18) The engine failed.

Boundedness: trangition bound

Event time: $M_{1}$ unifies with

moment([fail1])

Defining these three different relations of event time to temporal structure simplifies the computation of the ordering relations given by the perfect and non-perfect tenses.

\subsection{Temporal Ordering Relations}

The event time computed in Module 1 and the verb's tense and taxis comprise the input used in computing temporal ordering relations. Due to the pragmatic complexity of the perfect tenses and 
to space limitations, neither reference time nor taxis is discussed here (but cf. Passonneau, 1986a). The rules for the past and present tenses are quite simple. They locate the event time as coincident with or prior to the time of text production (i.e., the Report Time):

IF Tense=present

AND Taxis=non-perfect

THEN coincide(Event Time, Report Time)

IF Tense $=$ past

AND Taxis=non-perfect

THEN precedes(Event Time, Report Time)

These two rules in combination with the different relations of event time to the temporal structures of situations makes it possible to capture important facts about the interaction of tense and aspect. For example, present tense denotes an actual time only when applied to unbounded intervals. Thus a reference to an actual situation is computed for sentences like 19) but not 20).

19) The engine is failing.

20) The engine fails.

In 20), the present tense pertains not to a specific event of engine failure, but rather to the tendency for this type of situation to recur.

A predication denoting a past unbounded situation can be followed by a predication asserting the continuation or cessation of the same situation:

21) The pump was operating at 08:00 and is still operating.

A single interval would be generated for the two clauses in 21). However, a similar assertion following a predication with a transition event verb in the simple past is contradictory if still is interpreted as indicating persistence of the same event. ${ }^{16}$

22) ?The pump sheared the drive shaft and is still shearing it.

The event time for the first conjunct in 22) is a moment necessarily culminating in a new situation (i.e., a state of being sheared). Since the transition bound is dimensionless, the adverb still cannot refer to its persistence. A predication evoking an unspecified interval in a similar

\footnotetext{
15 Another reading of 22) refers to a uniqe event followed by iterations of the iame type of event.
}

context can be interpreted analogously to either 21) or 22):

23) The pump operated at 08:00 and is still operating.

The non-committal relation of event time to temporal structure for unspecified intervals makes both interpretations of 23) possible, and selecting among them is undoubtedly a pragmatic task rather than a semantic one. As we will see next, the utility of distinguishing between unbounded and unspecifled process predications is especially apparent in the context of temporal adverbials.

\section{Conclusion: Adverbial Modification}

The representations described above were inspired by remarks found in the literature on tense and aspect to the effect that the time sehemata (Vendler, 1987) associated with different situations are crucial to the way we perceive and talk about them. One of the crucial types of evidence used in deriving PUNDIT's temporal semantics as the interpretation of temporal adverbials in difierent contexts (Passonneau, 1986a). Consequently, one of the advantages to the representations is that they make it possible to tailor the interpretation of a temporal adverb to the temporal structure of the modified situation.

For example, specifying a different relation for the event time of an active interval, depending on its boundedness, yields different temporal relations between the situations described in sentences like 24-26), as shown informally in the examples.

24) The pump failed when the engine was rotating.

transition of failure during period of rotation

25) The pump failed when the engine rotated. transition of fallure during $O R$ at one endpoint of period of rotation

26) The engine rotated when the pump failed. Same as 25)

Sentences like 25) and 26) are often interpreted with the process (e.g., rotation) beginning at or after the transition event moment (e.g., failure). PUNDIT's representations of the temporal semantics of predications are explicit enough yet sufficiently non-committal to provide suitable input to a pragmatic reasoner that could decide these cases. 


\section{Acknowledgements}

I would like to thank Martha Palmer, Lynette Hirschman, Bonnie Webber and Debbje Dahl for their comments, encouragement and patience.

\section{REFERENCES}

Allen, James F. 1984. Towards a general theory of action and time. AI 23: 123-154.

Allen, James. F. 1983. Maintaining knowledge about temporal intervals. ACM 26.11:832-843.

Barwise, Jon and John Perry. 1983. Situations and Attitudes. Cambridge, Massachusetts: The MIT Press.

Dahl, Deborahl. 1986. Focusing and reference resolution in PUNDIT. Presented at AAAI. Philadelphia, PA.

Dahl, Deborah; Dowding, John; Hirschman, Lynette; Lang, Francois; Linebarger, Marcia; Palmer, Martha; Passonneau, Rebecca; Riley, Leslie. 1987. Integrating Syntax, Semantics, and Discourse: DARPA Natural Language Understanding Program. Final Report May, 1985-May, 1987.

Dowty, David R. 1986. The effects of aspectual class on the temporal structure of disconrse: semantics or pragmatics? Linguistics and Philosophy 9: 37-61.

Dowty, David R. 1979. Word Meaning and Montague Grammar. Dordrecht: D. Reidel.

Jakobson, Roman. 1971 [1957]. Shifters, verbal categories and the Russian verb. In his Selected Writings, Vol. 2, PP. 130-147. The Hague: Mouton.
Mourelatos, Alexander P. D. 1981. Events, processes, and states. In Tedeschi and Zaenen, pp. 191-212.

Palmer, Martha; Dahl, Deborah A.; Schiffman, Rebecca J. [Passonneau]; Hirschman, Lynette; Linebarger, Marcia; Dowding, John. 1986. Recovering Implicit Information. 24th Annual Meeting of the ACL. Columbia University, New York.

Passonneau, Rebecca. 1986a. A Computational Model of the Semantics of Tense and Aspect. Logic-Based Systems Technical Memo No. 43. Paoli Research Center. SDC. December, 1986.

Passonneau, Rebecca. 1986b. Designing Lexical Entries for a Limited Domain. Logic-Based Systems Technical Memo No. 42. Paoli Research Center. SDC. November, 1986.

Reichenbach, Hans. 1947. Elements of Symbolic Logic. New York: The Free Press.

Talmy, Leonard. 1985. Lexicalisation patterns. In Language Typology and Syntactic Description, vol. 3: Grammatical Categories and the Lexicon, pp. 57-151. Edited by Timothy Shopen. Cambridge: Cambridge University Press.

Taylor, Barry. 1977. Tense and continuity. Linguistics and Philosophy 1.

Tedeschi, P. J. and A. Zaenen, eds. 1981. Syntax and Semantics, vol 14: Tense and Aspect. New York: Academic Press.

Vendler, Zeno. 1967. Verbs and times. Linguistics in Philosophy. New York: Cornell University Press.

Vlach, Frank. 1981. The Semantics of the progressive. In Tedeschi and Zaenen, pp. 271-292. 